OPEN ACCESS

Edited by: Seppo Meri,

University of Helsinki, Finland

Reviewed by:

Arvind Sahu,

National Centre for Cell Science, India

Marcin Okrój,

Intercollegiate Faculty of Biotechnology of University of Gdańsk and Medical University of Gdańsk,

Poland

*Correspondence:

Richard J. H. Smith richard-smith@uiowa.edu

Specialty section:

This article was submitted to Molecular Innate Immunity, a section of the journal

Frontiers in Immunology

Received: 16 September 2020 Accepted: 05 November 2020 Published: 15 December 2020

Citation:

Zhang Y, Ghiringhelli Borsa N, Shao D, Dopler A, Jones MB, Meyer NC, Pitcher GR, Taylor $A O$, Nester $C M$, Schmidt CQ and Smith RJH (2020) Factor H Autoantibodies and Complement-Mediated Diseases. Front. Immunol. 11:607211. doi: 10.3389/fimmu.2020.607211

\section{Factor H Autoantibodies and Complement-Mediated Diseases}

\author{
Yuzhou Zhang ${ }^{1}$, Nicolo Ghiringhelli Borsa ${ }^{1}$, Dingwu Shao ${ }^{1}$, Arthur Dopler ${ }^{2}$, \\ Michael B. Jones ${ }^{1}$, Nicole C. Meyer ${ }^{1}$, Gabriella R. Pitcher ${ }^{1}$, Amanda O. Taylor ${ }^{1}$, \\ Carla M. Nester ${ }^{1}$, Christoph Q. Schmidt ${ }^{2}$ and Richard J. H. Smith ${ }^{1 *}$ \\ ${ }^{1}$ Molecular Otolaryngology and Renal Research Laboratories, University of lowa, lowa City, IA, United States, ${ }^{2}$ Institute of \\ Pharmacology of Natural Products \& Clinical Pharmacology, Ulm University, Ulm, Germany
}

Factor $\mathrm{H}(\mathrm{FH})$, a member of the regulators-of-complement-activation (RCA) family of proteins, circulates in human plasma at concentrations of $180-420 \mathrm{mg} / \mathrm{L}$ where it controls the alternative pathway (AP) of complement in the fluid phase and on cell surfaces. When the regulatory function of $\mathrm{FH}$ is impaired, complement-mediated tissue injury and inflammation occur, leading to diseases such as atypical hemolytic uremic syndrome (a thrombotic microangiopathy or TMA), C3 glomerulopathy (C3G) and monoclonal gammopathy of renal significance (MGRS). A pathophysiological cause of compromised $\mathrm{FH}$ function is the development of autoantibodies to various domains of the $\mathrm{FH}$ protein. $\mathrm{FH}$ autoantibodies (FHAAs) are identified in $10.9 \%$ of patients with aHUS, $3.2 \%$ of patients with C3G, and rarely in patients with MGRS. The phenotypic variability of FHAA-mediated disease reflects both the complexity of $\mathrm{FH}$ and the epitope specificity of FHAA for select regions of the native protein. In this paper, we have characterized FHAA epitopes in a large cohort of patients diagnosed with TMA, C3G or MGRS. We explore the epitopes recognized by FHAAs in these diseases and the association of FHAAs with the genetic deletion of both copies of the CFHR1 gene to show how these disease phenotypes are associated with this diverse spectrum of autoantibodies.

Keywords: factor H, autoantibodies, complement, C3 glomerulopathy, atypical hemolytic uremic syndrome, monoclonal gammopathy of renal significance

\section{INTRODUCTION}

Complement factor $\mathrm{H}$ (FH), a $155 \mathrm{KDa}$ glycoprotein comprised of 20 short consensus repeat (SCR) domains, circulates in the blood at concentrations of $180-420 \mathrm{mg} / \mathrm{L}$. It functions as the major regulator of the alternative pathway (AP) of complement in the fluid phase and on cell surfaces. Fluid-phase complement regulation is mediated by the four N-terminal SCRs of FH through two different mechanisms-decay accelerating activity (DAA) and co-factor activity (CA). DAA refers to the ability of $\mathrm{FH}$ to promote displacement of the $\mathrm{Bb}$ fragment of factor $\mathrm{B}(\mathrm{FB})$ off $\mathrm{C} 3$ convertase through SCR1-2, thereby accelerating the irreversible decay of $\mathrm{C} 3 \mathrm{bBb}$ to $\mathrm{C} 3 \mathrm{~b}$ and $\mathrm{Bb}$. CA refers to the role of FH as a facilitator of factor I (FI)-mediated proteolytic cleavage of $\mathrm{C} 3 \mathrm{~b}$ to an inactivated 
form of $\mathrm{C} 3 \mathrm{~b}$ called iC3b (1). In both scenarios, FH SCR1-4 interacts with the MG ring, CUB domain and TED domain on the $\mathrm{C} 3 \mathrm{~b}$ molecule (2). On cell surfaces, $\mathrm{FH}$ protects host surfaces from complement-mediated damage primarily through the two C-terminal SCRs, which recognize and bind to sialic acids, glycosaminoglycans (GAG), heparins, and a site on the C3bcleavage fragment $\mathrm{C} 3 \mathrm{~d}(3,4)$. These binding events ensure that the DAA function of FH is targeted to host cell surfaces, thereby protecting these cells from indiscriminate complement amplification that might otherwise be associated with surface deposition of C3b (5).

Genetic variation and/or acquired autoantibodies are the two major factors that impair FH function, and therefore are the primary drivers of two complement-mediated renal diseases, atypical hemolytic uremic syndrome (aHUS) and C3 glomerulopathy (C3G). The former, a type of thrombotic microangiopathy (TMA), is characterized by hemolytic anemia, thrombocytopenia and acute renal injury (6). TMA itself is an overarching term used to describe any condition characterized by thrombocytopenia and microangiopathic hemolytic anemia (MAHA) with varying degrees of organ damage in the setting of normal clotting parameters. Although tissue diagnosis, most commonly in the form of a kidney biopsy showing abnormalities in arterioles and capillaries with microvascular thrombosis, is required, TMA is often inferred from the observation of thrombocytopenia and MAHA in the appropriate clinical setting. Complement-mediated aHUS occurs primarily on host cell surfaces and leads to acute vascular endothelial injury and thrombosis. When left untreated, the likelihood of renal failure and mortality are high (7). The second disease associated with impaired $\mathrm{FH}$ function, $\mathrm{C} 3 \mathrm{G}$, is a chronic glomerulopathy characterized by predominant $\mathrm{C} 3$ deposition in renal glomeruli. Classic findings of glomerulonephritis (hematuria, proteinuria and variable degrees of renal impairment) result from fluid-phase complement dysregulation (8). Renal survival is about 10 years in up to $50 \%$ of affected individuals and following transplantation, approximately $50 \%$ of patients experience disease recurrence with allograft loss.

FHAAs have been identified as drivers of complement dysregulation in both aHUS and C3G (9). They are more common in aHUS, being detected in $\sim 10 \%$ of patients in European cohorts and up to 50\% in an Indian aHUS cohort (1012). In C3G cohorts, FHAAs are present in $~ 3 \%$ of patients $(13,14)$.

Interestingly, in aHUS, the presence of FHAAs is often associated with a common genetic variation known as a copy number variation $(\mathrm{CNV})$ in the $\mathrm{CFH}-\mathrm{CFHR}$ genomic region. The $\mathrm{CFH}$-CFHR gene family includes, in addition to $\mathrm{CFH}$, five complement factor H-related (CFHR) genes located directly 3' of the CFH gene in the order of CFHR3, CFHR1, CFHR4, CFHR2, and CFHR5. The CFHR genes arose as a result of genomic duplication and because of the high sequence homology, the region is prone to non-allelic homologous recombination, a process that can result in gene deletion, duplication and rearrangement.

Non-allelic homologous recombination gives rise to CNVs. Absence of both copies of CFHR1 due to homozygous deletion of CFHR3-CFHR1 (delCFHR3-1) or less commonly compound heterozygous deletion of CFHR3-CFHR1 and CFHR1-CFHR4 or homozygous deletion of CFHR1-CFHR4 is associated with an increased relative risk for aHUS as a consequence of the development of FHAAs, referred to as DEAP-HUS, DEficiency of CFHR1 plasma proteins and Autoantibody Positive Hemolytic Uremic Syndrome (15). It is important to note, however, that the increase in relative risk is small as homozygous delCFHR3-1 is common. About 3\% of European-Americans do not have any copies of CFHR3-CFHR1, a percentage that varies significantly by ethnic group $(16,17)$. The mechanism underlying the development of FHAAs in association with FHR1 deficiency is not well understood but may reflect structural differences between FHR1 and the carboxy terminus of FH (18). To date, there has been no such kind of association observed in C3G. In fact, FHAAs identified in C3G patients are frequently associated with the presence of $\mathrm{C} 3$ nephritic factors in children and with monoclonal gammopathy of renal significance (MGRS) in adults $(13,14,19,20)$.

Herein, we report the prevalence and immunological features of FHAAs in cohorts of aHUS and C3G patients from the USA.

\section{METHODS}

\section{Patients}

Patients with either C3G $(n=589)$ or aHUS $(n=448)$ referred to the Molecular Otolaryngology and Renal Research Laboratories (MORL) from 2013-'19 for FHAA testing were included in this study. Serum and plasma samples were collected using our standard operating procedure (SOP), aliquoted, and stored at $-80^{\circ} \mathrm{C}$ prior to testing (21). Control sera and plasma $(n=300)$ were collected using the same SOP. The study was approved by the Institutional Review Board of Carver College of Medicine at the University of Iowa.

\section{Anti-Factor H Autoantibody Assay}

FHAAs were detected as previously described (22, 23). Briefly, purified human FH (Complement Technology Inc, Tyler, TX) was coated in 1X PBS ( $\mathrm{pH}=7.4)$ at a concentration of $10 \mu \mathrm{g} / \mathrm{mL}$ on a 96-well micro-titer plate, which was then kept overnight at $4^{\circ} \mathrm{C}$. After washing three times with $1 \mathrm{X}$ PBST (1X PBS containing $0.1 \%$ Triton-X), free reactive sites were blocked with Ultrablock (AbD Serotec, Raleigh, NC) for $30 \mathrm{~min}$ at room temperature. Patient serum (1:50 dilution) was added for a 1-hour incubation at room temperature, after which plates were washed and incubated for another hour at room temperature with a horseradish peroxidase-labeled goat antihuman IgG antibody specific for the $\gamma$ chain. After final washings, enzymatic activity was measured using OPD (ophenylenediamine dihydrochloride) and absorbance was read at $\lambda 490$.

A standard curve (4-parameter logistic regression) was generated for each run by serial dilutions of a positive sample (aHUS49, 3,000 arbitrary units (AU) at 1:50). The value was calibrated to a positive sample kindly provided by Dr. Marie Agnès Dragon-Durey (Georges Pompidou hospital, Paris, France). 


\section{Epitope Mapping and Isotyping}

To map binding epitopes, recombinant FH fragments of SCRs1$6,6-8,8-15,15-18,18-20$ and mini-FH (1-4 and 19-20) were produced as previously described $(3,5,24)$, and used as capturing/coating proteins in the aforementioned protocol. Similarly, recombinant FHR1, 2, and 5 with 6X HIS tag were used as capturing/coating proteins for testing FHAA crossreactivity to FHR1, 2 or 5 .

To determine IgG subclass and light chains, the protocol was repeated with mouse anti-human IgG1, IgG2, IgG3, IgG4, kappa and lambda antibodies (all from Millipore Sigma) used at a dilution of 1:1,000-1:2,000 as detecting antibodies.

\section{Other Autoantibody Detection}

FB autoantibodies (FBAAs) were measured by ELISA and C3/ C4/C5 nephritic factors were measured by cell-based hemolytic methods as previously described $(25,26)$.

\section{M-Protein Detection}

All FHAA-positive patients were screened for M-proteins using serum protein electrophoresis and immunofixation electrophoresis (IFE) on a SPIFE Touch System (Helena Laboratories, Beaumont, TX).

\section{Complement Assays}

Serum levels of C3 were measured by ELISA (Hycult Biotech Inc., Uden, Netherlands). C4 was measured using radial immunodiffusion (The Binding Site Inc., Birmingham, UK). Soluble C5b-9 and FH levels were measured using ELISA kits (Quidel Corporation, San Diego, CA).

\section{Genetic Analysis}

Genomic DNA was extracted from peripheral blood using the Gentra Puregene Kit (Qiagen Inc., Valencia, CA) and integrity was evaluated by $1 \%$ agarose gel electrophoresis. Absorbance at 230:260:280 was measured using a NanoDrop 1000 spectrophotometer (Thermo Fisher Scientific, Wilmington, DE) to ensure DNA samples met quality metrics of 1.8 for $260 / 280$ and 260/230 ratios. DNA concentration was determined using the Qubit dsDNA HS Assay Kit (Life Technologies, Carlsbad, CA). Samples were then screened using the Genetic Renal Panel focused on complement gene abnormalities, as previously described (27). To interrogate the $C F H$-CFHR region, multiplex ligation-dependent probe amplification (MLPA) was performed using MRC Holland SALSA kit (Amsterdam, Netherlands) and in-house designed probes (28).

\section{Western Blotting for FHR1}

Serum or plasma (1:40 diluted) in Laemmli buffer was separated on $4-15 \%$ gel followed by in-house produced polyclonal rabbit antibodies to the first SCR of FHR1 and FHR2.

\section{Statistical Analyses}

Statistical analysis was performed using GraphPad (version 8.2). The Student $t$-test or Mann-Whitney $U$ Test was used to compare groups. $P<0.05$ was considered statistically significant.

\section{RESULTS}

\section{FHAAs in C3G and aHUS}

Nineteen patients (3.2\%) in the C3G cohort and 49 patients (10.9\%) in the aHUS cohort were positive for FHAAs. In both cohorts, both genders were equally affected (Tables 1 and 2), however FHAA-positive aHUS patients were significantly younger than FHAA-positive C3G patients (median age, 10.2 vs 38.3, respectively; $P<0.001$, Figure 1A). FHAA titers were also significantly higher in patients with aHUS as compared to patients with C3G (median, 4787 AU vs 1149 AU, respectively; $P<0.05$, Figure 1B).

\section{FHAA Targeting Epitopes and FHR1 Deficiency}

FHR1 deficiency as determined by either MLPA to detect homozygous deletion of the CFHR1 gene or Western blotting to detect absence of the FHR1 protein was observed in 37 of 49 (76\%) aHUS patients and 2 of 19 (11\%) C3G patients (Tables 3 and 4).

In aHUS, FHAA titers were higher in patients deficient as compared to patients replete in FHR1 (median 5,841 vs 3,217, respectively) although the difference was not statistically significant ( $P=0.131$, Mann-Whitney $U$ Test, Figure 1C). $37 / 37$ $(100 \%)$ of aHUS patients deficient in FHR1 carried FHAAs that primarily targeted the C-terminus of $\mathrm{FH}$. In four-fifths of these patients $(30 / 37,81 \%)$, the FHAAs cross-reacted with FHR1, while in one patient (aHUS31), cross-reactivity to FHR2 was also seen. In these patients, the addition of recombinant FHR1 blocked binding of FHAAs to FH. Multiple epitopes of FH were recognized in six of the 37 FHR1-deficient aHUS patients, including four patients who were co-positive for FHAAs against N-terminus SCRs and two patients who were copositive for FHAAs against mid-SCRs 8-15.

Of the 12 aHUS patients who express FHR1, six (aHUS41-46) had FHAAs that bind to the C-terminus of FH only, but in none of these patients did the FHAAs show cross-reactivity with FHR1. Two patients (aHUS38, 39) had FHAAs that bind to the N-terminus of FH only, while three patients (aHUS47-49) were co-positive for FHAAs that recognized $\mathrm{N}$ - and C-terminal epitopes of $\mathrm{FH}$. In two of these three patients, there was crossreactivity with FHR1. In one patient (aHUS40), FHAAs recognized an epitope in SCRs 8-15 of $\mathrm{FH}$.

Only two patients with $\mathrm{C} 3 \mathrm{G}$ were FHR1 deficient. In one patient (C3G1), FHAAs targeted the N-terminus (SCRs1-6) alone while in the other patient (C3G18), FHAAs reacted with the Cterminus of $\mathrm{FH}$ and also cross-reacted with FHR1. Of the 17 other C3G patients, all of whom express FHR1, 16 had FHAAs that recognized specific FH epitopes: four patients (C3G2-5) had FHAAs that bind to FH SCRs 1-6; two patients (C3G6, 7) had FHAAs that bind to FH SCRs 6-8; two patients (C3G8, 9) had FHAAs to both fragments; one patient (C3G10) had FHAAs that bind to FH SCRs 8-15; and four patients (C3G13-15, 17) had FHAAs that bind to SCRs 19 and 20. There were three patients (C3G12, 16, 19) whose FHAAs reacted with both $\mathrm{N}$ - and Cterminal SCRs of FH. 
TABLE 1 | Demographic and genetic data for aHUS patients.

\begin{tabular}{|c|c|c|c|c|c|c|c|}
\hline \multirow{2}{*}{$\begin{array}{l}\text { Patient } \\
\text { aHUS }\end{array}$} & \multirow[t]{2}{*}{ Sex } & \multirow[t]{2}{*}{ Age range } & \multirow[t]{2}{*}{ Ethnicity } & \multirow{2}{*}{$\frac{\text { Genetic findings }}{\text { (rare variant MAF<0.01\%) }}$} & \multicolumn{3}{|c|}{ Copies } \\
\hline & & & & & CFHR3 & CFHR1 & CFHR4 \\
\hline aHUS1 & $\mathrm{M}$ & $6-10$ & Caucasian & $\mathrm{N} / \mathrm{A}$ & N/A & N/A & $\mathrm{N} / \mathrm{A}$ \\
\hline aHUS2 & $\mathrm{M}$ & $6-10$ & Caucasian & no variants & 0 & 0 & 2 \\
\hline aHUS3 & $M$ & $11-15$ & Caucasian & no variants & 0 & 0 & 2 \\
\hline aHUS4 & $\mathrm{M}$ & $6-10$ & Caucasian & no variants & 1 & 0 & 1 \\
\hline aHUS5 & $\mathrm{F}$ & $6-10$ & Caucasian & N/A & 0 & 0 & 2 \\
\hline aHUS6 & $\mathrm{M}$ & $6-10$ & Caucasian & no variants & 0 & 0 & 2 \\
\hline aHUS7 & $\mathrm{F}$ & $16-20$ & Caucasian & no variants & 0 & 0 & 2 \\
\hline aHUS8 & $\mathrm{F}$ & $1-5$ & Caucasian & no variants & 0 & 0 & 2 \\
\hline aHUS9 & $\mathrm{F}$ & $6-10$ & Caucasian & N/A & $\mathrm{N} / \mathrm{A}$ & $\mathrm{N} / \mathrm{A}$ & $\mathrm{N} / \mathrm{A}$ \\
\hline aHUS10 & $\mathrm{M}$ & $16-20$ & African American & no variants & 0 & 0 & 2 \\
\hline aHUS11 & $M$ & $31-35$ & Caucasian & no variants & 0 & 0 & 2 \\
\hline aHUS12 & $\mathrm{F}$ & $6-10$ & Caucasian & no variants & 0 & 0 & 2 \\
\hline aHUS13 & M & $1-5$ & African American & CFHR5 c.427A>C, p.Thr143Pro & 0 & 0 & 2 \\
\hline aHUS14 & $\mathrm{F}$ & $6-10$ & Hispanic & no variants & 1 & 0 & 1 \\
\hline aHUS15 & M & $11-15$ & Caucasian & no variants & 0 & 0 & 2 \\
\hline aHUS16 & M & $11-15$ & Asian & no variants & 0 & 0 & 2 \\
\hline aHUS17 & $\mathrm{F}$ & $11-15$ & Hispanic & no variants & 0 & 0 & 2 \\
\hline aHUS18 & M & $6-10$ & Caucasian/African American & no variants & 0 & 0 & 2 \\
\hline aHUS19 & $\mathrm{F}$ & $6-10$ & African American & no variants & 0 & 0 & 2 \\
\hline aHUS20 & $\mathrm{M}$ & $6-10$ & Hispanic & N/A & $\mathrm{N} / \mathrm{A}$ & N/A & $\mathrm{N} / \mathrm{A}$ \\
\hline aHUS21 & $\mathrm{F}$ & $6-10$ & Caucasian & CFH c.3644G>A, p.Arg1215Gln & 0 & 0 & 2 \\
\hline aHUS22 & $\mathrm{M}$ & $11-15$ & Caucasian & no variants & 0 & 0 & 2 \\
\hline aHUS23 & M & $16-20$ & Caucasian & no variants & 0 & 0 & 2 \\
\hline aHUS24 & M & $61-65$ & Caucasian & no variants & 1 & 0 & 1 \\
\hline aHUS25 & $\mathrm{F}$ & $11-15$ & Caucasian & no variants & 0 & 0 & 2 \\
\hline aHUS26 & M & $11-15$ & Arabic & no variants & 0 & 0 & 2 \\
\hline aHUS27 & $M$ & $11-15$ & Caucasian & no variants & 1 & 0 & 1 \\
\hline aHUS28 & $M$ & $1-5$ & Caucasian & no variants & 0 & 0 & 2 \\
\hline aHUS29 & $\mathrm{F}$ & $11-15$ & Caucasian & no variants & 1 & 0 & 1 \\
\hline aHUS30 & $\mathrm{M}$ & $6-10$ & Hispanic & no variants & 0 & 0 & 2 \\
\hline aHUS31 & $\mathrm{F}$ & $6-10$ & Caucasian & no variants & 0 & 0 & 2 \\
\hline aHUS32 & $\mathrm{F}$ & $41-45$ & Caucasian & no variants & 1 & 0 & 1 \\
\hline aHUS33 & $\mathrm{F}$ & $16-20$ & African American & no variants & 0 & 0 & 2 \\
\hline aHUS34 & $\mathrm{F}$ & $6-10$ & Asian & no variants & 0 & 0 & 2 \\
\hline aHUS35 & $\mathrm{F}$ & $11-15$ & Asian & N/A & 0 & 0 & 2 \\
\hline aHUS36 & $M$ & $6-10$ & Hispanic & no variants & 0 & 0 & 2 \\
\hline aHUS37 & $M$ & $6-10$ & Caucasian & no variants & 0 & 0 & 2 \\
\hline aHUS38 & $\mathrm{M}$ & $1-5$ & African American & no variants & 1 & 1 & 2 \\
\hline aHUS39 & $M$ & $46-50$ & Caucasian & CFH c.3536T>C, p.lle1179Thr & 2 & 2 & 2 \\
\hline aHUS40 & $M$ & $51-55$ & Caucasian & no variants & 2 & 2 & 2 \\
\hline aHUS41 & M & $16-20$ & Hispanic & no variants & 2 & 2 & 2 \\
\hline aHUS42 & $\mathrm{F}$ & $6-10$ & Hispanic & no variants & 2 & 2 & 2 \\
\hline aHUS43 & $\mathrm{F}$ & $6-10$ & Caucasian & no variants & 2 & 2 & 2 \\
\hline aHUS44 & $\mathrm{F}$ & $11-15$ & Asian & no variants & 2 & 2 & 2 \\
\hline aHUS45 & $\mathrm{F}$ & $6-10$ & Hispanic & no variants & 2 & 1 & 1 \\
\hline aHUS46 & M & $71-75$ & Caucasian & no variants & 1 & 1 & 2 \\
\hline aHUS47 & $\mathrm{M}$ & $16-20$ & Caucasian & no variants & 2 & 2 & 2 \\
\hline aHUS48 & M & $1-5$ & Arabic & no variants & 2 & 2 & 2 \\
\hline aHUS49 & $\mathrm{F}$ & $1-5$ & Caucasian & no variants & $\mathrm{N} / \mathrm{A}$ & $\mathrm{N} / \mathrm{A}$ & $\mathrm{N} / \mathrm{A}$ \\
\hline
\end{tabular}

$N / A$, not available.

No patients in this study carried FHAAs that recognized FH SCRs15-18 or had cross reactivity to FHR5.

\section{IgG Subclasses and M-Proteins}

The distribution of IgG subclasses was similar in the two disease cohorts and is listed in Tables 3 and $\mathbf{4}$. In the aHUS cohort, the prominent subclass was IgG3 (35/49, 71\%), with most patients $(29 / 35,83 \%)$ having a restriction of either lambda or kappa, although six patients $(6 / 35,17 \%)$ were co-positive for lambda and kappa. Another six patients $(6 / 49,12 \%)$ were positive for only
IgG1 with either lambda or kappa restriction; two of these patients had MGRS (one each of IgG $\kappa$ and $\lambda$ ). Seven patients $(7 / 49,14 \%$ ) were co-positive for IgG1 and IgG3 and one patient $(1 / 49,2 \%)$ was co-positive for IgG1, IgG3 and IgG4. No patient was positive for IgG2. With respect to light chains, 25 patients $(25 / 49,51 \%)$ were positive for lambda only, 14 patients (14/49, 29\%) for kappa only, and 10 patients $(10 / 49,20 \%)$ for both.

In the $\mathrm{C} 3 \mathrm{G}$ cohort, the prominent subclass was also IgG3 with either lambda or kappa restriction $(11 / 19,58 \%)$. There were two patients with MGRS in this group (one each of $\operatorname{IgG} \kappa$ and $\lambda$ ). Five 
TABLE 2 | Demographic and genetic data for C3G patients.

\begin{tabular}{|c|c|c|c|c|c|c|c|}
\hline $\begin{array}{l}\text { Patient } \\
\text { C3G }\end{array}$ & Sex & Age range & Ethnicity & $\frac{\text { Genetic findings }}{\text { (rare variant } \mathrm{MAF}<0.01 \% \text { ) }}$ & \multicolumn{3}{|c|}{ Copies } \\
\hline C3G1 & M & $61-65$ & Caucasian & no variants & 1 & 0 & 1 \\
\hline C3G3 & $\mathrm{F}$ & $71-75$ & Caucasian & no variants & 2 & 2 & 2 \\
\hline C3G4 & M & $26-30$ & Caucasian & N/A & N/A & N/A & $\mathrm{N} / \mathrm{A}$ \\
\hline C3G5 & $\mathrm{F}$ & $71-75$ & Asian & C3 c.3214C>T, p.Arg1072Trp & 2 & 2 & 2 \\
\hline C3G8 & $\mathrm{F}$ & $21-25$ & Caucasian & no variants & 2 & 2 & 2 \\
\hline C3G9 & M & $36-40$ & Caucasian & no variants & 2 & 2 & 2 \\
\hline C3G10 & M & $81-85$ & Caucasian & no variants & 2 & 2 & 2 \\
\hline C3G11 & M & $66-70$ & Caucasian & no variants & 2 & 2 & 2 \\
\hline C3G12 & $\mathrm{F}$ & $61-65$ & Caucasian & no variants & 2 & 2 & 2 \\
\hline C3G13 & $\mathrm{F}$ & $6-10$ & Caucasian & CFH c.1854A>G, p.Asp619Gly & 2 & 2 & 2 \\
\hline C3G19 & $\mathrm{F}$ & $51-55$ & Caucasian & N/A & N/A & $\mathrm{N} / \mathrm{A}$ & $\mathrm{N} / \mathrm{A}$ \\
\hline
\end{tabular}

N/A, not available.

A

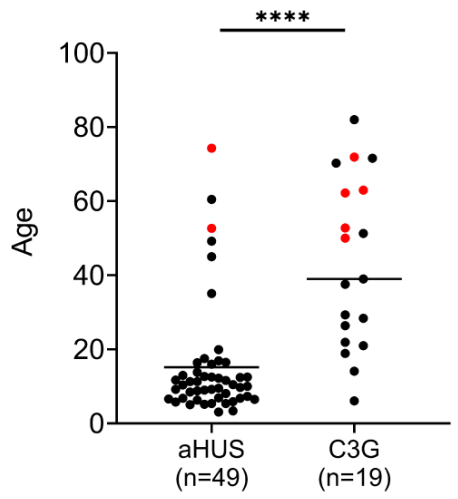

D

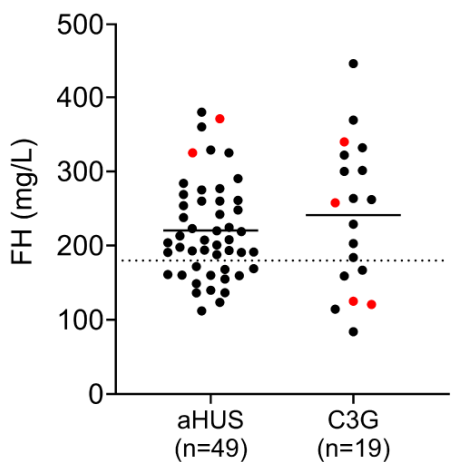

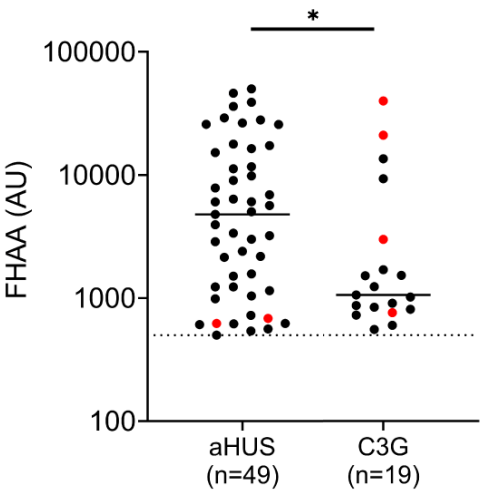

E

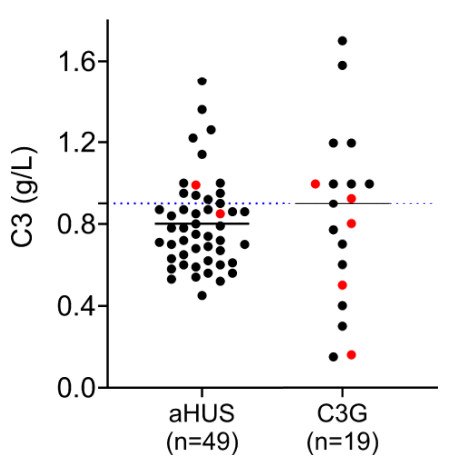

C

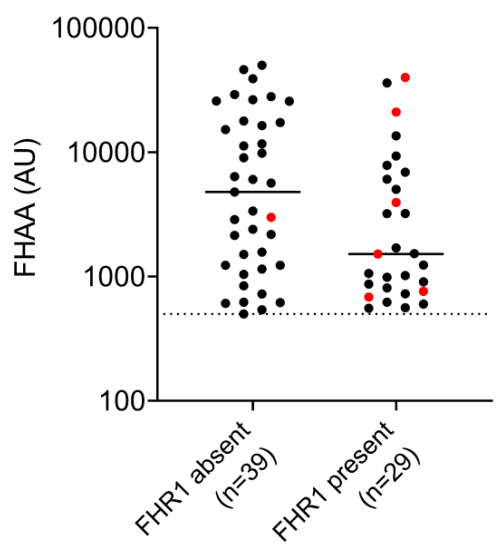

$\mathbf{F}$

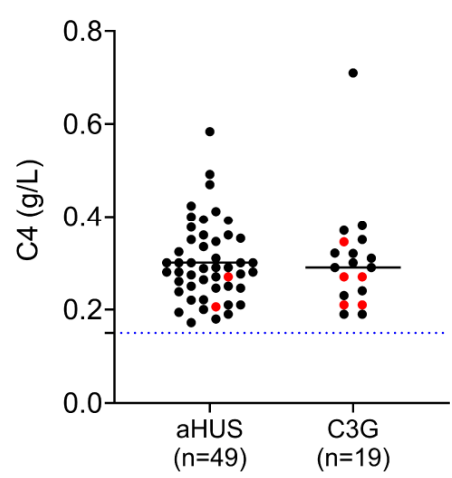

FIGURE 1 | FH autoantibodies (FHAAs) and key biomarkers at the age-of-onset of disease in patients with aHUS and C3G. Patient with M- proteins are in red. (A) Age; (B) FHAA titers in aHUS and C3G; (C) FHAAs (C3G and aHUS) with and without FHR1; (D) FH levels; (E) C3 levels; (F) C4 levels. Solid lines: medians. Dashed lines: normal cutoff based on results from 300 healthy individuals. ${ }^{*} P<0.05$ and ${ }^{\star \star \star \star} P<0.0001$ by Mann-Whitney $U$ test. 
TABLE 3 | FHAAs in patients with atypical hemolytic uremic syndrome.

\begin{tabular}{|c|c|c|c|c|c|c|c|c|c|c|c|c|c|}
\hline Patient & FHR1 $^{\wedge}$ & FHAA (AU) & \multicolumn{5}{|c|}{ FH Epitope } & Mini-FH & FHR1 & FHR2 & M-Spike & \multicolumn{2}{|c|}{ FHAA } \\
\hline aHUS1 & absent & 2875 & & & & & + & + & & & & $\lg G 3$ & $\lambda$ \\
\hline aHUS3 & absent & 540 & & & & & + & + & & & & $\lg G 3$ & k \\
\hline aHUS4 & absent & 9828 & & & & & + & + & & & & $\lg G 3$ & $\lambda$ \\
\hline aHUS5 & absent & 500 & & & & & + & + & & & & $\lg G 3$ & $\lambda$ \\
\hline aHUS8 & absent & 3362 & & & & & + & + & + & & & $\lg G 1$ & $\lambda$ \\
\hline aHUS9 & absent & 25690 & & & & & + & + & + & & & $\lg G 3$ & $\lambda$ \\
\hline aHUS10 & absent & 4787 & & & & & + & + & + & & & $\lg \mathrm{G} 1+\lg \mathrm{G} 3$ & k \\
\hline aHUS11 & absent & 2180 & & & & & + & $\mathrm{N} / \mathrm{D}$ & + & & & $\operatorname{lgG} 3$ & $\lambda$ \\
\hline aHUS12 & absent & 17290 & & & & & + & + & + & & & $\lg G 3$ & k \\
\hline aHUS13 & absent & 11220 & & & & & + & + & + & & & $\lg \mathrm{G} 1+\lg \mathrm{G} 3$ & $\lambda$ \\
\hline aHUS19 & absent & 39000 & & & & & + & + & + & & & $\lg G 3$ & k \\
\hline aHUS20 & absent & 1041 & & & & & + & $\mathrm{N} / \mathrm{D}$ & + & & & $\lg G 3$ & $\lambda+k$ \\
\hline aHUS21 & absent & 1148 & & & & & + & + & + & & & $\lg G 3$ & $\lambda$ \\
\hline aHUS22 & absent & 1230 & & & & & + & + & + & & & $\lg G 3$ & k \\
\hline aHUS23 & absent & 6041 & & & & & + & + & + & & & $\lg G 3$ & $\lambda$ \\
\hline aHUS24 & absent & 5640 & & & & & + & + & + & & & $\lg G 3$ & $\lambda$ \\
\hline aHUS25 & absent & 2401 & & & & & + & + & + & & & $\lg G 1$ & $\lambda$ \\
\hline aHUS26 & absent & 25750 & & & & & + & + & + & & & $\lg G 3$ & $\lambda+k$ \\
\hline aHUS27 & absent & 6361 & & & & & + & + & + & & & $\lg G 3$ & $\lambda$ \\
\hline aHUS28 & absent & 26450 & & & & & + & + & + & & & $\lg G 3$ & $\lambda$ \\
\hline aHUS29 & absent & 15220 & & & & & + & + & + & & & $\lg G 3$ & $\lambda$ \\
\hline aHUS30 & absent & 9018 & & & & & + & + & + & & & $\lg G 3$ & k \\
\hline aHUS40 & present & 3948 & & & + & & & & & & $\lg G \lambda$ & $\lg G 1$ & $\lambda$ \\
\hline aHUS41 & present & 5019 & & & & & + & + & & & & $\lg G 3$ & k \\
\hline aHUS42 & present & 35990 & & & & & + & + & & & & $\lg G 3$ & k \\
\hline aHUS43 & present & 562 & & & & & + & N/D & & & & $\lg G 3$ & k \\
\hline aHUS44 & present & 6895 & & & & & + & + & & & & $\lg G_{1}$ & k \\
\hline aHUS45 & present & 7823 & & & & & + & + & & & & $\lg G 3$ & k \\
\hline aHUS46 & present & 683 & & & & & + & + & & & $\lg G \mathrm{k}$ & $\lg \mathrm{G}_{1}$ & k \\
\hline aHUS47 & present & 986 & + & + & & & + & + & & & & $\lg G_{1}$ & k \\
\hline aHUS48 & present & 6053 & + & & & & + & + & + & & & $\lg G 1+\lg G 3$ & $\lambda+k$ \\
\hline aHUS49 & present & 3000 & + & + & & & + & + & + & & & $\lg G 3$ & $\lambda+k$ \\
\hline
\end{tabular}

ADetermined by MLPA or the Western if no DNAs.

Blank, Negative; N/D, not done.

patients $(5 / 19,26 \%)$ were positive for IgG1, with four patients showing lambda or kappa restriction, consistent with subclasses of M-spikes found in three patients (two $\operatorname{IgG} \kappa$ and one $\operatorname{IgG} \lambda$ ); one patient was positive for both light chains. One patient $(1 / 19$, $5 \%$ ) showed co-positivity for IgG1 and IgG3 with lambda restriction and one patient (5\%) showed co-positivity for IgG1 and IgG4 with reactivity to both light chains. One patient (1/19, $5 \%$ ) was positive for IgG4 only with kappa restriction. No patient was positive for IgG2.

\section{Other Acquired Drivers of Disease}

No other autoantibodies were detected in aHUS patients positive for FHAAs however one patient in the $\mathrm{C} 3 \mathrm{G}$ cohort was copositive for FBAA (C3G8) and two patients had C3 nephritic factors (C3G13, 18) (Table 4).

\section{Complement Dysregulation With FHAAs}

Low plasma FH levels were detected in 14 of 48 (29\%) and 6 of 19 (32\%) patients with aHUS and C3G, respectively (Figure 1D). 
TABLE 4 | FHAAs in patients with C3 glomerulopathy.

\begin{tabular}{|c|c|c|c|c|c|c|c|c|c|c|c|c|c|}
\hline \multirow[t]{2}{*}{ Patient } & \multirow[t]{2}{*}{$\mathrm{FHR}^{\wedge}$} & \multirow[t]{2}{*}{ FHAA (AU) } & \multicolumn{5}{|c|}{ FH Epitope } & \multirow[t]{2}{*}{ Mini-FH } & \multirow[t]{2}{*}{ FHR1 } & \multirow[t]{2}{*}{ FHR2 } & \multirow[t]{2}{*}{ M-Spike } & \multicolumn{2}{|c|}{ FHAA } \\
\hline & & & $1-6$ & $6-8$ & $8-15$ & $15-18$ & $18-20$ & & & & & IgG subclasses & Light chains \\
\hline C3G1 & absent & 2990 & + & & & & & + & & & $\lg G \mathrm{k}$ & $\lg G 1$ & k \\
\hline C3G2 & present & 9315 & + & & & & & + & & & & $\lg G 1+\lg G 4$ & $\lambda+\mathrm{k}$ \\
\hline C3G3 & present & 1059 & + & & & & & + & & & & $\operatorname{lgG} 4$ & $\lambda$ \\
\hline C3G4 & present & 13550 & + & & & & & + & & & & $\lg G 1+\lg G 3$ & $\lambda$ \\
\hline C3G5 & present & 21010 & + & & & & & + & & & $\operatorname{lgG} \mathrm{k}$ & $\lg G 1$ & $\mathrm{k}$ \\
\hline C3G6 & present & 1514 & & + & & & & & & & $\lg G \lambda$ & $\lg \mathrm{G} 1$ & $\lambda$ \\
\hline C3G7 & present & 39980 & & + & & & & & & & $\lg G \lambda$ & $\operatorname{lgG} 3$ & $\lambda$ \\
\hline C3G8 & present & 555 & + & + & & & & + & & & & $\operatorname{lgG} 3$ & k \\
\hline C3G9 & present & 809 & + & + & & & & + & & & & $\operatorname{lgG} 3$ & k \\
\hline C3G10 & present & 1017 & & & + & & & & & & & $\lg \mathrm{g} 1$ & $\lambda$ \\
\hline C3G11 & present & 869 & + & + & + & & + & + & & & & $\lg G 1$ & $\lambda+\mathrm{k}$ \\
\hline C3G12 & present & 759 & + & + & & & + & + & & & $\operatorname{lgG~k}$ & $\operatorname{lgG3}$ & k \\
\hline C3G13* & present & 1525 & & & & & + & + & & & & $\operatorname{lgG} 3$ & $\mathrm{k}$ \\
\hline C3G14 & present & 601 & & & & & + & + & & & & $\operatorname{lgG3}$ & k \\
\hline C3G15 & present & 725 & & & & & + & + & & & & $\operatorname{lgG} 3$ & $\lambda$ \\
\hline C3G16 & present & 1697 & & + & & & + & + & + & + & & lgG3 & $\lambda$ \\
\hline C3G17 & present & 1238 & & & & & + & + & + & + & & $\operatorname{lgG} 3$ & k \\
\hline C3G18* & absent & 843 & & & & & + & N/D & + & & & IgG3 & $\mathrm{k}$ \\
\hline C3G19 & present & 908 & + & & & & + & + & + & & & $\operatorname{lgG} 3$ & k \\
\hline
\end{tabular}

'Determined by MLPA or the Western if no DNAs.

${ }^{*} \mathrm{C} 3 \mathrm{G} 13$ and C3G18 are positive for C3Nefs.

${ }^{\#} \mathrm{C} 3 \mathrm{G} 8$ is also positive for FBAAs.

Blank, negative; N/D, not done.

There was no correlation between FH levels and FHAA titers. C3 levels were low in 12 of $20(60 \%)$ patients with aHUS and 7 of 16 (44\%) patients with C3G (Figure 1E). C4 levels were normal in all patients (Figure 1F). Soluble C5b-9 was elevated in 15 of 19 patients (79\%) with aHUS not on Eculizumab and in 11 of 16 C3G patients (also not on Eculizumab; 69\%), consistent with uncontrolled activity of the terminal complement pathway.

\section{Genetic Findings}

Genetic testing was completed in 45 aHUS and 15 C3G patients. Ultra-rare genetic variants (defined as a minor allele frequency (MAF) $<0.01 \%$ and resulting in a nonsynonymous amino acid change) were identified in five patients (Tables 1 and 2). Three patients carry rare variants in the $C F H$ gene (patients aHUS21, 39 and C3G13). The variants found in the two aHUS patients were in SCR20 of FH. One, FH p.R1215Q (in aHUS21), affects surface regulation by impairing surface heparin binding $(3,29)$; for the second variant, FH p.Ile1179Thr (in aHUS39), functional data are unavailable. The C3G patient (C3G13) carries FH p.Asp619Gly in SCR10, again a variant for which functional data are lacking. Another C3G patient (C3G5) carries C3 p.Arg1072Trp. In addition, one aHUS patient (aHUS13) carries a rare variant of unknown significance in the CFHR5 gene.

\section{DISCUSSION}

Herein, we report a retrospective study of FHAAs in aHUS and C3G patient cohorts from North America. Overall, FHAAs were found in $10.9 \%$ of aHUS and $3.2 \%$ of C3G patients, respectively, consistent with prior reports in populations of European decent $(13,30)$. The high prevalence of FHR1 deficiency in association with FHAAs is seen only in the aHUS cohort and not in the C3G cohort (Tables 3 and $\mathbf{4}$ ).

In more than $80 \%$ of patients with FHR1 deficiency, FHAAs bind to both the carboxy-terminus of FH and FHR1. In these patients, FHAA titers tend to be extremely high in the acute phase of disease (Figure 1B, data collected in acute phase) but drop during remission, suggesting that the presence of FHR1 plays an important role in suppressing auto-immunogenicity of $\mathrm{FH}$ when a trigger is present. Consistent with this hypothesis, in these patients recombinant FHR1 can compete off $\mathrm{FH}$ for FHAA binding.

The last two SCRs of FH are essential for self-surface recognition and have ligand-binding sites for heparan sulfate, sialic acid and the complement cleavage product, C3d. Importantly, sequence homology between SCRs 19 and 20 of FH and SCRs 4 and 5 of FHR1 is very high, with amino acid differences only at two positions (S1191 and V1197 on FH vs L290 and A296 on FHR1) (31). It has been postulated that subtle conformational changes at residues 1,182-1,189 (due to S1191) in FH SCR20 occur during infections and may be auto antigenic in the absence of FHR1 (18). Our data appear to support this hypothesis since $>92 \%$ of FHAA patients with FHR1 deficiency were pediatric cases with a median age of 10 (IQR 6.9-12.7), suggesting that the development of FHAAs is associated with common school-related infections.

The consequence of FHAAs that impair C-terminal function of $\mathrm{FH}$ is dysregulation of complement control on host cell surfaces. FHR1 deficiency alone, however, is not sufficient to trigger the generation of FHAAs. The deletion of $79.4 \mathrm{~kb}$ on 
chromosome 1 (gnomAD ID: MCNV_1_81) that includes two $\mathrm{CFH}$-related genes, $\mathrm{CFHR} 3$ and $C F H R 1$, is a common $\mathrm{CNV}$ in the human genome with homozygous deletion of both copies of the CFHR3-CFHR1 genes present in $4.1 \%$ of Europeans, $16.2 \%$ of Africans, $1.9 \%$ of Latinos and $0.3 \%$ of East Asians (data based on the reported non-diploid $\mathrm{CN}$ frequency in the gnom $\mathrm{AD}$ ). In fact, FHR1 deficiency has been reported in FHAA-negative aHUS patients at a frequency that is higher than that in an ethnically matched control population (32).

In our aHUS cohort, in contrast, after excluding patients with FHAAs, the frequency of FHR1 deficiency was comparable to that found in a control population of European decent (nine of 255 patients, 3.5\%). In addition, we commonly detected FHAAs in patients who express CFHR1. Of the 29 patients with FHR1 who were positive for FHAAs, there were 12 cases of aHUS ( $24 \%$ of all FHAA-positive cases of aHUS) and 17 cases of C3G (89\% of all FHAA-positive cases of $\mathrm{C} 3 \mathrm{G}$ ). In about half of these cases, the antibody recognized carboxy-terminal SCRs of $\mathrm{FH}$; however, in no case was there cross-reactivity to FHR1 and the addition of recombinant FHR1 ex vivo has no influence on FHAA binding results.

In seven of 19 (37\%) C3G patients (C3G1-5, 8, 9) and two of 49 (4\%) aHUS patients (aHUS38, 39) with FHR1, the FHAAs reacted only with SCRs at the N-terminus (SCRs1-6). Epitope reactivity was confirmed using mini-FH (SCRs1-4+19-20). The N-terminal SCRs1-4 is the site of DAA and CA. Blocking these two major regulatory functions of $\mathrm{FH}$ would be predicted to lead to complement dysregulation in the fluid phase as well as on cell surfaces. Why some patients develop a C3G phenotype and others develop an aHUS phenotype is not clear but may reflect differences in the degree of residual DAA or CA, as well as factors that determine complement control in local microenvironments like the glomerulus. We speculate that in general $\mathrm{C} 3 \mathrm{G}$ patients have better control of AP activity on cell surfaces as compared to aHUS patients due to the presence of complement regulators (i.e. CD46, $\mathrm{CD} 55)$ or due to $\mathrm{FH}$ itself. In this study, for example, we identified two aHUS patients (aHUS21, 39) carrying pathogenic or likely pathogenic variants in $\mathrm{CFH}$ as genetic drivers of disease. With ongoing dysregulation primarily in the fluid phase, C3G patients present with chronic phenotypes such as proteinuria and hematuria, while aHUS patients present with acute phenotypes like endothelial cell damage and complement-mediated coagulopathy.

None of the aHUS patients had other acquired drivers of disease while in C3G, two patients had C3Nefs and one patient had FBAAs $(P<0.05$ by Fisher exact test). This finding suggests that while aHUS patients are more likely to have FHAAs as a sole acquired driver, C3G patients may be co-positive for other acquired drivers of disease such as nephritic factors, perhaps implying that FHAAs play a secondary role in $\mathrm{C} 3 \mathrm{G}$.

Interestingly, in 37 of 49 (76\%) aHUS patients and 16 of 19 (84\%) C3G patients, the circulating FHAAs demonstrated monoclonal characteristics (one subclass of heavy chains + one type of light chains) with IgG3 followed by IgG1 being the most common heavy chain isotypes. These data are consistent with most previous reports $(9,33-35)$, but are at odds with the findings

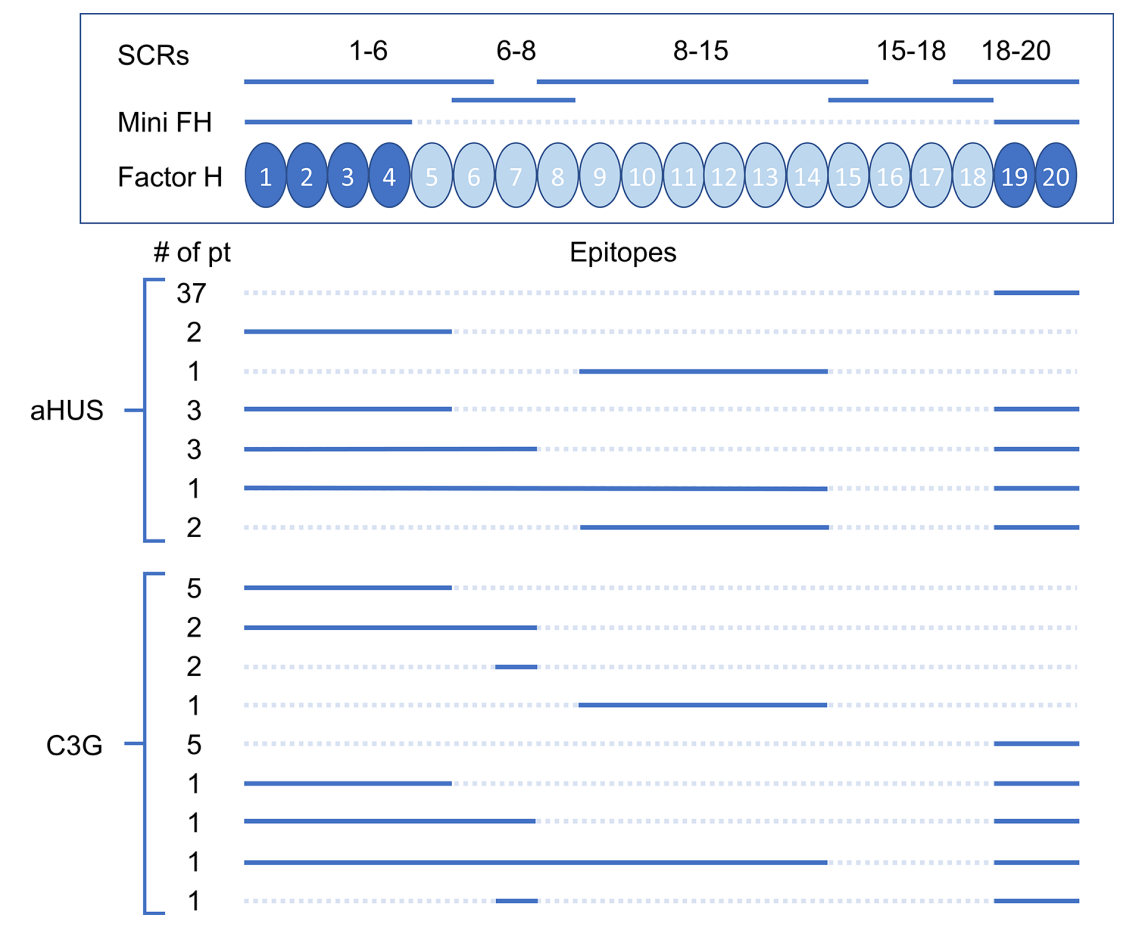

FIGURE 2 | Epitopes of factor $\mathrm{H}$ autoantibodies (FHAAs) targeting domains on factor $\mathrm{H}$ (FH). Recombinant FH fragments (SCRs) and the mini FH construct are shown above a schematic of FH. Below are shown the epitope mapping results for aHUS $(n=49)$ and C3G $(n=19)$ patients (solid lines = positive FHAA results, dash lines = negative FHAA results) 
described by Guo et al., who showed that FHAAs in the Chinese population are typically polyclonal (36). The presence of the lambda light chain was more dominant in the aHUS cohort while both light chains had equal presence in the C3G cohort.

Albeit many FHAAs appeared monoclonal, we only observed seven M-proteins by IFE (the most sensitive method for detecting circulating M-proteins) in both cohorts, two aHUS patients (aHUS40, 46) and five C3G patients (C3G1, 5-7, 12). These patients were all over 50 years of age and while monoclonal gammopathy has emerged as an underlying cause of C3G in the elderly $(13,14,37)$, its association with aHUS has not been previously reported.

MGRS is a recently defined disease entity, in which the underlying pathogenesis for the renal disease is associated with circulating monoclonal immunoglobulins or M-proteins that drive renal injury. The malignant clonal $\mathrm{B}$ cell clones do not meet criteria for overt multiple myeloma/B-cell proliferation. Mproteins can directly deposit in the glomeruli and activate complement through the classical pathway resulting in immune complex glomerulonephritis, or alternatively directly activate the $\mathrm{AP}$ in the fluid phase or block complement regulators resulting in M-protein induced C3G or aHUS (13, 14, 19, 20).

As compared to $\mathrm{C} 3 \mathrm{G}(5 / 19,26 \%)$, the prevalence of $\mathrm{M}$ proteins in aHUS $(2 / 49,4 \%)$ is rare. Patient aHUS46 has a monoclonal IgG1 kappa directed against the C-terminus of $\mathrm{FH}$ (without FHR1 reactivity) that impairs the surface regulation of the AP, consistent with aHUS phenotype. However, in patient aHUS40, FHAAs (also IgG1 but lambda light chain) only bind to the mid portion of $\mathrm{FH}$ (SCRs 8-15). In this patient, plasma $\mathrm{FH}$ is normal, C3 is borderline low, and sC5b-9 is slightly elevated, findings consistent with ongoing complement dysregulation. Interestingly, C3G patient C3G10 is also positive for FHAAs that target only SCRs 8-15 of FH with no apparent M-proteins and has a similar biomarker profile (normal $\mathrm{FH}$, borderline low C3, slightly elevated sC5b-9). Additional research is warranted in these two cases, as it would be of great interest to clarify the underlying mechanisms by which FHAAs that target the midportion of $\mathrm{FH}$ impact complement control.

Finally, two $\mathrm{C} 3 \mathrm{G}$ patients $(\mathrm{C} 3 \mathrm{G} 6,7)$ circulate monoclonal FHAAs (IgG1, kappa and lambda, respectively) that target only SCR7 of FH. Recently, Li, et al. described a Chinese C3G patient with FHAAs that bind to SCR7. Functional studies showed that in this patient, the SCR7-recognizing FHAAs inhibited FH binding to $\mathrm{C} 3 \mathrm{~b}$ and accelerated formation of $\mathrm{C} 3$ convertase (37). In addition, FHL-1, a truncated version of FH containing the first 7 SCRs of $\mathrm{FH}$, has regulatory activity that involves domains SCRs5-7 (38). Thus, compromising SCR7 function may play a role in the pathogenesis of MGRS-C3G.

\section{REFERENCES}

1. Rodriguez de Cordoba S, Esparza-Gordillo J, Goicoechea de Jorge E, LopezTrascasa M, Sanchez-Corral P. The human complement factor H: functional roles, genetic variations and disease associations. Mol Immunol (2004) 41 (4):355-67. doi: 10.1016/j.molimm.2004.02.005

2. Wu J, Wu YQ, Ricklin D, Janssen BJ, Lambris JD, Gros P. Structure of complement fragment $\mathrm{C} 3 \mathrm{~b}$-factor $\mathrm{H}$ and implications for host protection by
In conclusion, we provide a comprehensive analysis of FHAAs in patients with aHUS and C3G (Figure 2). In aHUS, the absence of FHR1 is associated with a high incidence of FHAAs in patients age under 20 years of age; in patients over 50 years of age, FHAAs may be associated with MGRS. In C3G, FHAAs are more likely to be co-positive with other autoantibodies and the likelihood of MGRS in older patients is higher. Our data highlight the value of epitope mapping and isotyping in patients who are positive for FHAAs as a method of refining the underlying pathophysiology of complement dysregulation in the fluid phase and/or on cell surfaces.

\section{DATA AVAILABILITY STATEMENT}

The datasets generated for this study are available on request to the corresponding author.

\section{ETHICS STATEMENT}

The studies involving human participants were reviewed and approved by Institutional Review Board of Carver College of Medicine at the University of Iowa. Written informed consent to participate in this study was provided by the participants' legal guardian/next of kin.

\section{AUTHOR CONTRIBUTIONS}

$\mathrm{YZ}$ and RS designed the research, analyzed and interpreted data, and wrote the manuscript. NG, DS, AD, MJ, NM, GP, and AT performed experiments and participated in data analysis. $\mathrm{CN}$ and CS provided crucial conceptual input. CS contributed essential reagents. All authors contributed to the article and approved the submitted version.

\section{FUNDING}

Supported in part by National Institutes of Health R01 DK110023.

\section{ACKNOWLEDGMENTS}

We thank those patients with C3G and aHUS whose participation made this research possible.

complement regulators. Nat Immunol (2009) 10(7):728-33. doi: 10.1038/ ni. 1755

3. Morgan HP, Schmidt CQ, Guariento M, Blaum BS, Gillespie D, Herbert $\mathrm{AP}$, et al. Structural basis for engagement by complement factor $\mathrm{H}$ of $\mathrm{C} 3 \mathrm{~b}$ on a self surface. Nat Struct Mol Biol (2011) 18(4):463-70. doi: 10.1038/ nsmb. 2018

4. Schramm EC, Roumenina LT, Rybkine T, Chauvet S, Vieira-Martins P, Hue C, et al. Mapping interactions between complement $\mathrm{C} 3$ and regulators using 
mutations in atypical hemolytic uremic syndrome. Blood (2015) 125(15):235969. doi: 10.1182/blood-2014-10-609073

5. Schmidt CQ, Herbert AP, Kavanagh D, Gandy C, Fenton CJ, Blaum BS, et al. A new map of glycosaminoglycan and $\mathrm{C} 3 \mathrm{~b}$ binding sites on factor $\mathrm{H}$. J Immunol (2008) 181(4):2610-9. doi: 10.4049/jimmunol.181.4.2610

6. Hofer J, Giner T, Jozsi M. Complement factor H-antibody-associated hemolytic uremic syndrome: pathogenesis, clinical presentation, and treatment. Semin Thromb Hemost (2014) 40(4):431-43. doi: 10.1055/s-0034-1375297

7. Noris M, Remuzzi G. Atypical hemolytic-uremic syndrome. N Engl J Med (2009) 361(17):1676-87. doi: 10.1056/NEJMra0902814

8. Smith RJH, Appel GB, Blom AM, Cook HT, D’Agati VD, Fakhouri F, et al. C3 glomerulopathy - understanding a rare complement-driven renal disease. Nat Rev Nephrol (2019) 15(3):129-43. doi: 10.1038/s41581-018-0107-2

9. Blanc C, Roumenina LT, Ashraf Y, Hyvarinen S, Sethi SK, Ranchin B, et al. Overall neutralization of complement factor $\mathrm{H}$ by autoantibodies in the acute phase of the autoimmune form of atypical hemolytic uremic syndrome. J Immunol (2012) 189(7):3528-37. doi: 10.4049/jimmunol.1200679

10. Dragon-Durey MA, Sethi SK, Bagga A, Blanc C, Blouin J, Ranchin B, et al. Clinical features of anti-factor $\mathrm{H}$ autoantibody-associated hemolytic uremic syndrome. J Am Soc Nephrol (2010) 21(12):2180-7. doi: 10.1681/ASN.2010030315

11. Moore I, Strain L, Pappworth I, Kavanagh D, Barlow PN, Herbert AP, et al. Association of factor $\mathrm{H}$ autoantibodies with deletions of CFHR1, CFHR3, CFHR4, and with mutations in CFH, CFI, CD46, and C3 in patients with atypical hemolytic uremic syndrome. Blood (2010) 115(2):379-87. doi: 10.1182/blood-2009-05-221549

12. Sinha A, Gulati A, Saini S, Blanc C, Gupta A, Gurjar BS, et al. Prompt plasma exchanges and immunosuppressive treatment improves the outcomes of antifactor $\mathrm{H}$ autoantibody-associated hemolytic uremic syndrome in children. Kidney Int (2014) 85(5):1151-60. doi: 10.1038/ki.2013.373

13. Blanc C, Togarsimalemath SK, Chauvet S, Le Quintrec M, Moulin B, Buchler M, et al. Anti-factor $\mathrm{H}$ autoantibodies in $\mathrm{C} 3$ glomerulopathies and in atypical hemolytic uremic syndrome: one target, two diseases. J Immunol (2015) 194 (11):5129-38. doi: 10.4049/jimmunol.1402770

14. Goodship TH, Pappworth IY, Toth T, Denton M, Houlberg K, McCormick F, et al. Factor $\mathrm{H}$ autoantibodies in membranoproliferative glomerulonephritis. Mol Immunol (2012) 52(3-4):200-6. doi: 10.1016/j.molimm.2012.05.009

15. Zipfel PF, Mache C, Muller D, Licht C, Wigger M, Skerka C, et al. DEAP-HUS: deficiency of CFHR plasma proteins and autoantibody-positive form of hemolytic uremic syndrome. Pediatr Nephrol (2010) 25(10):2009-19. doi: 10.1007/s00467-010-1446-9

16. Leban N, Abarrategui-Garrido C, Fariza-Requejo E, Aminoso-Carbonero C, Pinto S, Chibani JB, et al. Factor $\mathrm{H}$ and CFHR1 polymorphisms associated with atypical Haemolytic Uraemic Syndrome (aHUS) are differently expressed in Tunisian and in Caucasian populations. Int J Immunogenet (2012) 39 (2):110-3. doi: 10.1111/j.1744-313X.2011.01071.x

17. Fremeaux-Bacchi V, Fakhouri F, Garnier A, Bienaime F, Dragon-Durey MA, Ngo S, et al. Genetics and outcome of atypical hemolytic uremic syndrome: a nationwide French series comparing children and adults. Clin J Am Soc Nephrol (2013) 8(4):554-62. doi: 10.2215/CJN.04760512

18. Bhattacharjee A, Reuter S, Trojnar E, Kolodziejczyk R, Seeberger H, Hyvarinen S, et al. The major autoantibody epitope on factor $\mathrm{H}$ in atypical hemolytic uremic syndrome is structurally different from its homologous site in factor H-related protein 1, supporting a novel model for induction of autoimmunity in this disease. J Biol Chem (2015) 290(15):9500-10. doi: 10.1074/jbc.M114.630871

19. Zand L, Kattah A, Fervenza FC, Smith RJ, Nasr SH, Zhang Y, et al. C3 glomerulonephritis associated with monoclonal gammopathy: a case series. Am J Kidney Dis (2013) 62(3):506-14. doi: 10.1053/j.ajkd.2013.02.370

20. Sethi S, Sukov WR, Zhang Y, Fervenza FC, Lager DJ, Miller DV, et al. Dense deposit disease associated with monoclonal gammopathy of undetermined significance. Am J Kidney Dis (2010) 56(5):977-82. doi: 10.1053/j.ajkd.2010.06.021

21. Zhang Y, Nester CM, Martin B, Skjoedt MO, Meyer NC, Shao D, et al. Defining the complement biomarker profile of C3 glomerulopathy. Clin J Am Soc Nephrol (2014) 9(11):1876-82. doi: 10.2215/CJN.01820214

22. Watson R, Lindner S, Bordereau P, Hunze EM, Tak F, Ngo S, et al. Standardisation of the factor $\mathrm{H}$ autoantibody assay. Immunobiology (2014) 219(1):9-16. doi: 10.1016/j.imbio.2013.06.004

23. Dragon-Durey MA, Loirat C, Cloarec S, Macher MA, Blouin J, Nivet H, et al. Anti-Factor $\mathrm{H}$ autoantibodies associated with atypical hemolytic uremic syndrome. J Am Soc Nephrol (2005) 16(2):555-63. doi: 10.1681/ ASN.2004050380

24. Schmidt CQ, Bai H, Lin Z, Risitano AM, Barlow PN, Ricklin D, et al. Rational engineering of a minimized immune inhibitor with unique triple-targeting properties. J Immunol (2013) 190(11):5712-21. doi: 10.4049/jimmunol.1203548

25. Zhang Y, Meyer NC, Wang K, Nishimura C, Frees K, Jones M, et al. Causes of alternative pathway dysregulation in dense deposit disease. Clin J Am Soc Nephrol (2012) 7(2):265-74. doi: 10.2215/CJN.07900811

26. Zhang Y, Meyer NC, Fervenza FC, Lau W, Keenan A, Cara-Fuentes G, et al. C4 Nephritic Factors in C3 Glomerulopathy: A Case Series. Am J Kidney Dis (2017) 70(6):834-43. doi: 10.1053/j.ajkd.2017.07.004

27. Bu F, Borsa NG, Jones MB, Takanami E, Nishimura C, Hauer JJ, et al. HighThroughput Genetic Testing for Thrombotic Microangiopathies and C3 Glomerulopathies. J Am Soc Nephrol (2016) 27(4):1245-53. doi: 10.1681/ ASN.2015040385

28. Bu F, Zhang Y, Wang K, Borsa NG, Jones MB, Taylor AO, et al. Genetic Analysis of 400 Patients Refines Understanding and Implicates a New Gene in Atypical Hemolytic Uremic Syndrome. J Am Soc Nephrol (2018) 29(12):280919. doi: 10.1681/ASN.2018070759

29. Lehtinen MJ, Rops AL, Isenman DE, van der Vlag J, Jokiranta TS. Mutations of factor $\mathrm{H}$ impair regulation of surface-bound C3b by three mechanisms in atypical hemolytic uremic syndrome. J Biol Chem (2009) 284(23):15650-8. doi: $10.1074 /$ jbc.M900814200

30. Valoti E, Alberti M, Iatropoulos P, Piras R, Mele C, Breno M, et al. Rare Functional Variants in Complement Genes and Anti-FH Autoantibodies-Associated aHUS. Front Immunol (2019) 10:853. doi: 10.3389/fimmu.2019.00853

31. Zipfel PF, Wiech T, Stea ED, Skerka C. CFHR Gene Variations Provide Insights in the Pathogenesis of the Kidney Diseases Atypical Hemolytic Uremic Syndrome and C3 Glomerulopathy. J Am Soc Nephrol (2020) 31 (2):241-56. doi: 10.1681/ASN.2019050515

32. Gurjar BS, Manikanta Sriharsha T, Bhasym A, Prabhu S, Puraswani M, Khandelwal $\mathrm{P}$, et al. Characterization of genetic predisposition and autoantibody profile in atypical haemolytic-uraemic syndrome. Immunology (2018) 154(4):663-72. doi: 10.1111/imm.12916

33. Strobel S, Abarrategui-Garrido C, Fariza-Requejo E, Seeberger H, SanchezCorral P, Jozsi M. Factor H-related protein 1 neutralizes anti-factor $\mathrm{H}$ autoantibodies in autoimmune hemolytic uremic syndrome. Kidney Int (2011) 80(4):397-404. doi: 10.1038/ki.2011.152

34. Durey MA, Sinha A, Togarsimalemath SK, Bagga A. Anti-complement-factor H-associated glomerulopathies. Nat Rev Nephrol (2016) 12(9):563-78. doi: 10.1038/nrneph.2016.99

35. Nozal P, Bernabeu-Herrero ME, Uzonyi B, Szilagyi A, Hyvarinen S, Prohaszka Z, et al. Heterogeneity but individual constancy of epitopes, isotypes and avidity of factor $\mathrm{H}$ autoantibodies in atypical hemolytic uremic syndrome. Mol Immunol (2016) 70:47-55. doi: 10.1016/j.molimm.2015.12.005

36. Guo WY, Song D, Liu XR, Chen Z, Xiao HJ, Ding J, et al. Immunological features and functional analysis of anti-CFH autoantibodies in patients with atypical hemolytic uremic syndrome. Pediatr Nephrol (2019) 34(2):269-81. doi: 10.1007/s00467-018-4074-4

37. Li LL, Li ZY, Wang SX, Yu XJ, Tan Y, Wang Y, et al. Monoclonal immunoglobulin mediates complement activation in monoclonal gammopathy associated-C3 glomerulonephritis. BMC Nephrol (2019) 20 (1):459. doi: 10.1186/s12882-019-1640-3

38. Dopler A, Guntau L, Harder MJ, Palmer A, Hochsmann B, Schrezenmeier H, et al. Self versus Nonself Discrimination by the Soluble Complement Regulators Factor $\mathrm{H}$ and FHL-1. J Immunol (2019) 202(7):2082-94. doi: 10.4049/jimmunol.1801545

Conflict of Interest: The authors declare that the research was conducted in the absence of any commercial or financial relationships that could be construed as a potential conflict of interest.

Copyright (๑ 2020 Zhang, Ghiringhelli Borsa, Shao, Dopler, Jones, Meyer, Pitcher, Taylor, Nester, Schmidt and Smith. This is an open-access article distributed under the terms of the Creative Commons Attribution License (CC BY). The use, distribution or reproduction in other forums is permitted, provided the original author(s) and the copyright owner(s) are credited and that the original publication in this journal is cited, in accordance with accepted academic practice. No use, distribution or reproduction is permitted which does not comply with these terms. 